\title{
ON ABSOLUTELY SEGREGATED ALGEBRAS AND RELATIVE 3-COHOMOLOGY GROUPS OF AN ALGEBRA
}

\author{
TADASI NAKAYAMA
}

Recently M. Ikeda [1] succeeded in determining the structure of absolutely segregated algebras, i.e. algebras whose 2-cohomology groups all vanish. His beautiful result reads: an algebra $A$, of finite rank over its ground field, is absolutely segregated if and only if i) the residue-algebra $A / N$ modulo the radical $N$ is separable and, moreover, ii) the $A$-left-module $N$ is an $\left(M_{0}\right)$-module. A. simplification was given by $\mathrm{H}$. Nagao [5], who obtained, besides an interesting result on algebras with vanishing 3 -(or higher) cohomology groups, an elegant short proof to the fact that under the assumption of i), the property ii) is necessary, and sufficient, for the absolute segregation of $A^{2}{ }^{2}$

In the present paper we shall show that, in an absolutely segregated algebra $A$, not only the radical $N$, but every left-ideal of $A$ is an $\left(M_{0}\right)$-left-module of $A$ (and every right-ideal of $A$ is an $\left(M_{0}\right)$-right-module of $A$ ). Besides that this property of absolutely segregated algebras is rather striking, as it seems to the writer, our approach to it, which we shall present in the followings, seems to be of some interest for itself, as there come into context a certain modification of cohomology groups (which we may call relative cohomology groups) and certain 3-cocycles (in the modified, relative sense), yielding thus a new significance of 3-cohomology. ${ }^{3)}$ We want also to note that, although our method is closely related with Nagao's one, we start independently of the condition i). Further, our approach is free from the finiteness of algebras and can be applied to general algebras of infinite rank with vanishing 2-cohomology groups, to yield some informations about them, though we have to leave, in this paper, their complete struciural characterization open.

Received July 10, 1953.

1) A left-module $\mathfrak{m}$ of an algebra $A$ is called an $\left(M_{0}\right)$-module, if it has the following property: if a left-module $\mathfrak{M}$ of $A$ contains an $A$-submodule $\mathfrak{n}$ such that $\mathfrak{M} / \mathfrak{n}$ is $A$-isomorphic to $\mathfrak{m}$, then $\mathfrak{M}$ possesses a second $A$-submodule $\mathfrak{m}^{\prime}$ (necessarily $A$-isomorphic to $\mathfrak{m}$,) such that $\mathfrak{M}$ is the direct sum $\mathfrak{M}=\mathfrak{m}^{\prime} \oplus \mathfrak{r}$. For its structural characterization: Cf. Nagao-Nakayama [6].

2) Once this is shown, also the necessity of i) can easily be shown by Ikeda's argument of ground field extension.

3) This significance of our $\mathfrak{l}$-relative 3 -cohomology is in a sense similar to the significance of "obstruction 3-cocycles" of Hochschild [3]. but is different. 
The writer wishes to thank H. Nagao and M. Ikeda for their friendly cooperation for the present work.

\section{§1. A modified cohomology theory}

Let $A$ be an algebra, of finite or infinite rank, over a field $\Omega$. Let $\mathfrak{l}$ be a. left-ideal of $A$, and consider an $A$-double-module m satisfying

$$
\mathrm{ml}=0 \text {. }
$$

Denote by $C_{\mathfrak{l}}^{l}(A, \mathrm{~m})$ the group (in fact an $\Omega$-module) of those $n$-cochains $f$ of $A$ in $m$, which possess the property: $f\left(a_{1}, a_{2}, \ldots, a_{n}\right)$ depend only on $a_{1}, a_{2}$, $\ldots, a_{n-1}$ and on the residue-class of $a_{n}$ modulo $\mathfrak{l}$, or, what amounts to the same thing, $f\left(a_{1}, a_{2}, \ldots, a_{n}\right)=0$ whenever $a_{n} \in \mathbb{l}$.

We verify readily that the coboundary operation $\delta$ maps $C_{\mathfrak{l}}^{n}(A, \mathfrak{m})$ into the similarly defined subgroup $C_{\mathfrak{l}}^{n+1}(A, \mathfrak{m})$ of the $(n+1)$-cochain group $C^{n+1}(A, \mathfrak{m})$. Denoting by $Z_{\mathfrak{l}}^{n}(A, \mathfrak{m})$ the intersection of $C_{\mathfrak{l}}^{n}(A, \mathfrak{m})$ with the group $Z^{n}(A, \mathfrak{m})$ of $n$-cocycles, we get thus a residue-group

$$
H_{\mathfrak{l}}^{n}(A, \mathfrak{m})=Z_{\mathfrak{l}}^{n}(A, \mathfrak{m}) / \delta C_{\mathfrak{l}}^{n-1}(A, \mathfrak{m})
$$

which we wish to call the l-relative $n$-cohomology group of $A$ in $\mathrm{m}$ (an $A$-doublemodule satisfying (1)); we shall also speak of -relative cochains and I-relative cocycles.

(More generally, we may consider, besides our $\mathfrak{l}$, a series of two-sided) ideals $z_{1}, z_{2}, \ldots$ of $A$ satisfying

$$
\mathfrak{l} \supseteqq z_{1} \supseteqq z_{\varepsilon} \supseteqq \ldots,
$$

and confine our consideration to those $n$-cochains and $n$-cocycles which depend only on the residue-class of the last argument modulo $\mathfrak{l}$, the residue-class of the last-but-one argument modulo $z_{1}$, the residue-class of the last-but-two argument modulo $z_{2}$, and so on, to get a further modified cohomology group. And Hochschild's [1] reduction theorem, for instance, can be given in such generalized setting. But in this note we shall confine ourselved to the above -relative cohomology theory which is sufficient for our present purpose.)

After Hochschild [1] we consider the 1-cochain group $C^{1}(A, \mathrm{~m})$ as an $A$ double-module, setting

$$
\begin{aligned}
& (a f)\left(a^{\prime}\right)=a f\left(a^{\prime}\right), \\
& (f a)\left(a^{\prime}\right)=f\left(a a^{\prime}\right)-f(a) a^{\prime}
\end{aligned}
$$

for $f \in C^{1}(A, m)$ and $a, a^{\prime} \in A$. We see readily that, with $\mathfrak{l}$ satisfying (1), the submodule $C_{\mathfrak{l}}^{1}(A, \mathrm{~m})$ is $A$-(two-sided-)allowable. (Moreover, it satisfies the condition (1), with $\mathfrak{m}$ replaced by $C_{\mathfrak{l}}^{\prime}(A, \mathfrak{m})$ (i.e. we have $C_{\mathfrak{l}}^{1}(A, \mathfrak{m}) \mathfrak{l}=0$ ) if $\mathfrak{l}$ is (not only a left-ideal but) a (two-sided) ideal of $A$.)

Now, let $n \geqslant 1$. With an $n$-cochain $f$ in $C_{\mathfrak{l}}^{n}(A, \mathfrak{m})$ we associate an (ordinary) 
$(n-1)$-cochain $\bar{f}$ of in the A-double-module $C_{l}^{1}(A, m)$ such as

$$
\bar{f}\left(a_{1}, a_{2}, \ldots, a_{n-1}\right)\left(a_{n}\right)=f\left(a_{1}, a_{2}, \ldots, a_{n}\right) .
$$

Conversely, for every element $\bar{f}$ of $C^{n-1}\left(A, C_{\mathrm{I}}^{1}(A, \mathrm{~m})\right)$ we get by (3) an element $f$ of $C_{\mathfrak{l}}^{n}(A, \mathfrak{m})$ with which our $\bar{f}$ is associated. Thus we have

$$
C_{l}^{n}(A, \mathfrak{m}) \cong C^{n-1}\left(A, C_{l}^{1}(\mathrm{~A}, \mathrm{~m})\right) \quad(n \geqslant 1)
$$

This correspondence is in harmony with the coboundary operation. Hence we have

$$
Z_{l}^{2}(A, \mathfrak{m}) \cong Z^{n-1}\left(A, C_{\mathfrak{l}}^{1}(A, \mathrm{~m})\right) \quad(n \cong 1)
$$

and further

$$
H_{\mathfrak{l}}^{n}(A, \mathfrak{m}) \cong H^{n-1}\left(A, C_{\mathfrak{l}}^{1}(A, \mathfrak{m})\right) \quad(n \geqslant 2)
$$

which is Hochschild's reduction theorem in our generalized formulation.

\section{§2. A significance of the (-relative 3-cohomology}

Let, again, $A$ be an algebra, of finite or infinite rank, over a field $\Omega$, and let $t$ be a left-ideal of $A$. Take an $\Omega$-submodule $A^{0}$ of $A$ (which is in general neither a subring nor a left-ideal) such that is the direct sum of $A^{0}$ and $\mathfrak{l}$ :

$$
A=A^{0} \oplus \text { r. }
$$

With an element $a$ of $A$, we denote by $a^{0}$ the uniquely determined element of $A$ satisfing $a \equiv a^{0} \bmod$ l. Put, for $a, b \in A$,

$$
b a=(b a)^{0}+\lambda(b, a), \quad \lambda(b, a) \in \mathfrak{l} .
$$

Considering the product $c b a$ we get

$$
\lambda(c b, a)=\lambda\left(c,(b a)^{0}\right)+c \lambda(b, a) .
$$

Further

$$
\lambda(b, l)=b l \quad(l \in l) .
$$

Now, let $\mathfrak{R}$ be an $A$-left-module and $m$ its $A$-(left-) submodule. Take an $\Omega$-submodule $\mathfrak{M}^{1}$ of $\mathfrak{M}$ such that, similarly as above,

$$
\mathfrak{M}=\mathfrak{M}^{1} \oplus \mathbb{m}
$$

Denote the representative in $\mathfrak{M}^{1}$ of the class modulo $\mathfrak{m}$ of an element $u$ of $\mathfrak{M}$ by $u^{1}$, and put, with $a \in A$,

$$
a u=(a u)^{1}+\mu(a, u), \quad \mu(a, u) \in m .
$$

We have

$$
\mu(b a, u)=\mu\left(b,(a u)^{1}\right)+b \mu(a, u),
$$


for $a, b A$, and

$$
\mu(a, m)=a m \quad(m \in m) .
$$

We assume now that the residue-module $\mathfrak{M} / \mathfrak{m}$ is $A$-(left-)isomorphic to our left-ideal $\mathfrak{l}$. For $l \in \mathfrak{l}$ we denote by $l^{\prime}$ the element of $\mathfrak{M}^{1}$ whose class rnodulo $m$ corresponds to $l$ by the (once fixed) isomorphism, and we write, for the sake of brevity, $\mu(a, l)$ in place of $\mu\left(a, l^{\prime}\right)$.

On considering $\mathrm{m}$ as an (not only $A$-left but) $A$-double-module by setting

$$
\mathrm{m} A=0 \text {, }
$$

we put

$$
f(c, b, a)=\mu\left(c, \lambda\left(b, a^{0}\right)\right),
$$

to get an element of $C^{3}(A, \mathfrak{m})$, in fact of $C_{\mathfrak{l}}^{3}(A, \mathfrak{m})$. We have

$$
\begin{aligned}
(\delta f)(d, & c, b, a) \\
= & d \mu\left(c, \lambda\left(b, a^{0}\right)\right)-\mu\left(d c, \lambda\left(b, a^{0}\right)\right) \\
& +\mu\left(d, \lambda\left(c b, a^{0}\right)\right)-\mu\left(d, \lambda\left(c,(b a)^{0}\right)\right) \\
= & d \mu\left(c, \lambda\left(b, a^{0}\right)\right)-\mu\left(d, c \lambda\left(b, a^{0}\right)\right)-d \mu\left(c, \lambda\left(b, a^{0}\right)\right) \\
& +\mu\left(d, \lambda\left(c,\left(b a^{0}\right)^{0}\right)+\mu\left(d, c \lambda\left(b, a^{0}\right)\right)-\mu\left(d, \lambda\left(c,(b a)^{0}\right)\right)\right. \\
= & 0
\end{aligned}
$$

by (15), (9), (13) (and the bilinearity of $\lambda, \mu)$; observe that $\left(b a^{0}\right)^{0}=(b a)^{0}$ (as $r$ is a left-ideal). Thus

$$
f(\text { defined in }(16)) \in Z_{\mathrm{l}}^{3}(A, \mathrm{~m}) .
$$

Now assume that there exists $h \in C_{I}^{2}(A, \mathrm{~m})$ satisfying

$$
\delta h=f \text {, }
$$

i.e.

$$
\mu\left(c, \lambda\left(b, a^{0}\right)\right)=c h(b, a)-h(c b, a)+h(c, b a) .
$$

With this -relative 2 -cochain $h$ we put

$$
\nu(b, a)=-h(b, a)+\mu\left(b, a-a^{0}\right)
$$

$(a, b \in A)$. Then, as $h \in C_{\mathfrak{l}}^{2}(A, \mathfrak{m})$,

$$
\nu(b, l)=\mu(b, l) \quad(l \in \mathfrak{l}) .
$$

Further

$$
\begin{aligned}
c \nu(b, a) & =-c h(b, a)+c \mu\left(b, a-a^{0}\right), \\
\nu(c b, a) & =-h(c b, a)+\mu\left(c b, a-a^{0}\right) \\
& =-h(c b, a)+\mu\left(c, b\left(a-a^{0}\right)\right)+c \mu\left(b, a-a^{0}\right), \\
\nu(c, b a) & =\nu\left(c,(b a)^{0}+\lambda(b, a)\right) \\
& =-h(c, b a)+\mu(c, \lambda(b, a)),
\end{aligned}
$$


by (13) and (8), whence

$$
\begin{aligned}
c \nu(b, a) & -\nu(c b, a)+\nu(c, b a) \\
= & -c h(b, a)+h(c b, a)-\mu\left(c, b\left(a-a^{0}\right)\right) \\
& -h(c, b a)+\mu(c, \lambda(b, a)) \\
= & -\mu\left(c, \lambda\left(b, a^{0}\right)\right)+\mu(c, \lambda(b, a))-\mu\left(c, b\left(a-a^{0}\right)\right) \\
= & \mu\left(c,-\lambda(b, a)+\lambda(b, a)-b\left(a-a^{0}\right)\right)
\end{aligned}
$$

by $\left(18^{\prime}\right)$. Here the second argument is 0 by virtue of (10). Thus

$$
c_{\nu}(b, a)-\nu((c b, a)+\nu(c, b a)=0,
$$

for every $a, b, c \in A$.

Introduce now an 2 -module $A \S$-isomorphic to $A$, by a correspondence $a \hookleftarrow a^{\S}$, and set

$$
\mathscr{P}=A s \oplus m \text {. }
$$

We define

$$
b\left(a^{\natural}+m\right)=(b a) s+1(b, a)+b m
$$

$\left(a^{\rho} \in A^{\beta}, \quad m \in m\right)$. Then the relation (21) assures that $A$ becomes an $A$-leftmodule. Clearly the residue-module $A / m$ is $A$-(left $)$ isomorphic to the $A$-lefmodule $A$. On the other hand, because of $(20)$, the $A$-(left-) submodule $r \$+m$ is $A$-(left-)isomorphic to $D i$. where $1 \$$ is the image of $i$ by our $(\Omega-)$ isomorphism of $A$ and $A^{\S}$.

Further, for $u \in \mathfrak{I}$ we denote by $\alpha(u)$ the (counter-)image in $A$ of the class modulo $m$ of $u$, thus $\alpha(u) \in A$ and

$$
\alpha(u) \S \equiv u \bmod . \mathrm{m} \text {. }
$$

For $u, v \in \mathfrak{A}$ we define the product $u v$ to be the image of $v$ by the operation $\alpha(u)$ :

$$
u v=\alpha(u) v
$$

Then

$$
\begin{aligned}
u(v w) & =u(\alpha(v) w)=\alpha(u)(\alpha(v) w)=(\alpha(u) \alpha(v)) w \\
& =(\alpha(\alpha(u) v)) w=(u v) w
\end{aligned}
$$

because of the $A$-left-isomorphism of $A$ and $\mathfrak{Y} / \mathfrak{m}$, among others. So $\mathfrak{i}$ becomes an algebra, over 2 , and $A$ and $\mathfrak{N} / \mathrm{m}$ are isomorphic also as algebras.

This prove the "if" part of the following theorem, whose "only if" part can be shown simply by reversing the above argument: ${ }^{4)}$

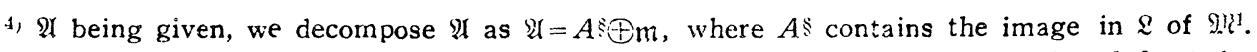
Define $n(b, a)$ by the case $m=0$ of $(22)$. Then we get $(20)$ and $(21)$. Define $h(b, a)$ by (19). We can verify (18') by virtue of (13) and (10). 
TheOREm $10^{5)}$ Let $\mathfrak{l}$ be a left-ideal of an algebra $A$. Let $\mathfrak{M}$ be a left-module of $A, \mathfrak{m}$ an $A$-(left-)submodule of $\mathfrak{M}$, such that $\mathfrak{M} / \mathrm{m}$ is $A$-(left $)$ isomorphic to 1. Define $f \in Z_{\mathrm{I}}^{3}(A, \mathfrak{m})$ by ((8), (12) and) (16) (where we put $\mathfrak{m} A=0$ (15)). If, and only if, the class of $f$ in $H_{\mathfrak{l}}^{3}(A, \mathfrak{m})$ is 0 , there exists an algebra 2 such that 1) II is an extension of $A$ with the kernel $\mathfrak{m}$ (i.e. I contains $\mathfrak{m}$ as an ideal, the residue-algebra $\mathfrak{A} / \mathfrak{m}$ is isomorphic to $A$, and the left and right operations on $\mathrm{m}$ of the elements of $\mathfrak{U}$ coincide with the corresponding operations of their images in $A$ ) (where we set again $m A=0$ (whence $m \mathfrak{A}=0)$ ), 2) if $\mathbb{2}$ is the leftideal of $\mathfrak{M}$ containing $\mathrm{m}$ such that $\mathfrak{I} / \mathrm{m}$ corresponds to $\mathfrak{l}$ by the isomorphism of $\mathfrak{Y} / \mathrm{m}$ and $A$, then the $\mathfrak{Y} / \mathrm{m}$-left-module ${ }^{6} \mathfrak{L}$ and the A-left-module $\mathfrak{M}$ are operatorisomorphic of second kind (i.e. semilinear-isomorphic) for our isomorphism of II/m and $A$.

Needless to say that the "if" part of our theorem is certainly applicable when we have

$$
H_{\mathfrak{l}}^{3}(A, \mathfrak{m})=0 .
$$

\section{§3. Algebras with vanishing 2-cohomology groups}

Let $A$ be, again, an algebra of finite or infinite rank over a field $\Omega$, and suppose now that the (ordinary) 2-cohomology group of $A$ in any $A$-doublemodule is $0 .^{7)}$ Then, because of the reduction theorem (6), we have certainly (25). With our $A$, let $\mathfrak{l}, \mathfrak{M}$ and $\mathfrak{m}$ be as in Theorem 1 . There exists, by virtue of Theorem 1, an extension $\mathfrak{U}$ of $A$, with kernel $\mathfrak{m}$, satisfying, besides 1 ), the condition 2) there. As further $H^{2}(A, \mathfrak{m})=0, \mathfrak{M}$ possesses a subalgebra $\widetilde{A}$ such that

$$
\mathfrak{P}=\tilde{A} \oplus \mathfrak{m} \text {. }
$$

$\tilde{A}$ is evidently isomorphic to $A$. Let $\widetilde{\mathfrak{l}}$ be the image, by this isomorphism, of our left-ideal $\mathfrak{l}$ of $A$. Then clearly the left-ideal $\mathfrak{L}$ of $\mathfrak{A}$ (defined in Theorem 1 ) is the direct sum of $\tilde{I}$ and $m$ :

$$
\mathfrak{L}=\tilde{\mathfrak{l}} \oplus \mathfrak{m}
$$

As $\mathfrak{m} \mathfrak{A}=0$, the left-ideal $\tilde{\mathfrak{I}}$ of $\tilde{\mathfrak{A}}$ is also a left-ideal of $\mathfrak{H}$, and (27) is a direct decomposition of $\mathfrak{Q}$ into left-subideals $\widetilde{\mathfrak{l}}$ and $\mathfrak{m}$. In view of the property 2 ) (in Theorem 1), this entails a direct decomposition of $\mathfrak{M}$ into $\mathfrak{m}$ and a second $A$ -

5) See Footnote 3 ).

6) Observe that ( $\mathfrak{m} \mathscr{A}=0$ whence) $\mathfrak{m} \mathfrak{R}=0$.

i) This is equivalent to that $A$ is segregated in every extension with nilpotent kernel; we may restrict ourselves to singular extensions, i.e. extensions with kernel ${ }^{*}$ satisfying $\mathfrak{t}^{2}=0$. If further $A$ is of finite rank, over $\Omega$, then $A$ is "absolutely" segregated in the sense that $A$ is segregated in every extension of finite rank ([1]). As, however, we are dealing with algebras of possibly infinite rank, it is perhaps not appropriate to call our $A$ (with absolutely vanishing 2-cohomology group) absolutely segregated. 
left-submodule, say $\tilde{\mathrm{m}}$,

$$
\mathfrak{M}=\tilde{m} \oplus \mathfrak{m} .
$$

Since we have started with an arbitrary $A$-left-module $\mathfrak{M}$ possessing a residuemodule $\mathfrak{M} / \mathrm{m} A$-isomorphic to $\mathfrak{l}$, this shows that $\mathfrak{l}$ is, as an $A$-left-module, an $\left(M_{0}\right)$-module. Thus

THEOREM 2. Let $A$ be an algebra (of finite or infinite rank over the ground field) whose 2-cohomology groups all vanish. Then every left-ideal 1 of $A$ is an $\left(M_{0}\right)$-left-module of $A$ and every right-ideal $\mathfrak{r}$ of $A$ is an $\left(M_{0}\right)$-right-module of $A$.

This theorem is free from any finiteness assumption. Assume now that our $A$ satisfies minimum and maximum conditions for left-ideals and that $A$ (or, more generally, its residue-algebra modulo the radical) is algebraic (over the ground field). Let $N$ be the radical of $A$. We assert that the center of any simple component of $A / N$ is separable over the ground field, say $\Omega$ as before. We needs

LemMA. Let an algebra $A$ with vanishing 2-cohomology groups satisfy minimum and maximum conditions for left-ideals. Let $e$ be a primitive idempotent element in $A$. Then $e A e$ is a sfield, or, what is the same, $e N e=0$, where $N$ is the radical of $A$.

Proof. As the assumption and the conclusion remain unaffected by adjunction of an identity, we may, and shall, assume that $A$ has an identity element 1. Let $1=\sum_{x=1}^{k} e_{\kappa}$ be a decomposition of 1 into a sum of mutually orthogonal primitive idempotent elements. We may assume, without loss in generality, that $e_{1}, e_{2}, \ldots, e_{k}$ are so enumerated that with a certain $h(\leqq k)$

i) $A e_{1}, A e_{2}, \ldots, A e_{h}$ are mutually non-isomorphic,

ii) every $A e_{\kappa}$ is isomorphic to one (and only one) of $A e_{1}, A e_{2}, \ldots, A e_{h}$, and

iii) $\left[A e_{1}\right] \leqq\left[A e_{2}\right] \leqq \ldots \leqq\left[A e_{h}\right]$, where $\left[A e_{\kappa}\right]$ denotes the composition-length of the left-ideal $A e_{\mathrm{k}}$.

We may suppose further that $e$ is among $e_{1}, e_{2}, \ldots, e_{h}$. Now, each direct summand $N e_{\mathrm{\kappa}}$ of $N$ is, together with $N$, an $\left(M_{0}\right)$-(left-)module of $A$. By [6], Theorem 1 (cf. "Remark" there), $N e_{\kappa}$ is thus directly decomposed into a number of left-subideals of $A$ each of which is isomorphic to a left-ideal $A e_{\lambda}(\lambda=1,2$, $\ldots, h)$. Let $t_{\lambda_{\kappa}}$ be the number of such components of $N e_{\kappa}$ isomorphic to $A e_{\lambda}$. We have

$$
\left[A e_{\kappa}\right]-1=\left[N e_{\kappa}\right]=\sum_{\Lambda=1}^{h} t_{\lambda \kappa}\left[A e_{\kappa}\right] .
$$

Thus $t_{\lambda \kappa}=0$ whenever $\lambda \geqq \kappa(\kappa, \lambda=1,2, \ldots, h)$. We want to show that every composition-residule-module of $N e_{\kappa}$ is isomorphic to a module $A e_{\lambda} / N e_{\lambda}$ with $\lambda<\kappa$ ( which entails certainly $e_{\kappa} N e_{\kappa}=0$ ). Suppose that this is proved for indices younger 
than $\kappa$. The composition-residue-modules of $A e_{\kappa}$ are those of $N e_{\lambda}$, each repeated $t_{\lambda \kappa}$ times, and the modules $A e_{\lambda} / N e_{\lambda}$, again repeated $t_{\lambda_{k}}$ times. Here only $\lambda^{\prime}$ s with $\lambda<\kappa$ come into consideration ( since $t_{\lambda \kappa}=0$ for $\lambda \geqslant \kappa$ ). It is clear then, by virtue of our induction assumption, that the composition-residue-modules are isomorphic to some $A e_{\lambda} / N e_{\kappa}$ with $\lambda<\kappa$.

THEOREM 3. Let $A$ be an algebraic algebra over a field 2 , which satisfies minimum and maximum conditions for left-ideals, and let $N$ be the radical of A. If the 2-cohomology groups of $A$ all vanish, then the center of each simple componeni of the semisimple algebra $A / N$ is separable over $\Omega$.

Proof. Suppose that the center of a simple component of $A / N$ is inseparable over $\Omega$, contrary to the assertion. There exists then, as one readily sees, a finite (inseparable) extension $A$ of $\Omega$ such that the algebra $A$ has a primitive idempotent element $e$ with $e M e \neq 0$, where $M$ is the radical of $A$. On the other hand, $A_{\mathrm{A}}$ has, together with $A$, vanishing 2-cohomology groups. ${ }^{8)}$ Thus we have a contradiction, in view of our Lemma.

Considering, finally, an algebra of finite rank, we have the following refinement of rkeda's result.

THEOREM 4. Let $A$ be an algebra of finile rank over a field \& possessing $a$ unit element. If all the 2-cohomology groups of $A$ vanish (or, what is the same, $A$ is absolutely segregated), then the semisimple residue-cigebra $A / N$ of $A$ modulo the radical $N$ is separable and every direcily indecomposable left-(right-) ideal of $A$ is (A-left-)(right-)ismorphic to a left-(right-)ideal direct component of $A, I f$, conversely, $A / N$ is separable and every directly indecomposable direct component of the left-ideal $N$ is isomorphic to a direct sum of A-left-subideals $A$-isomorphic to left-ideal direct components of $A$, then $A$ is absolutely segregated.

Proof. The first half is clear from what we have shown. As for the (easier) second half, we shall give a rephrasement (which is not at all an improvement) of Nagao's [5] proof to it. Assume namely that $A / N$ is separable and that $N$ is an $\left(M_{0}\right)$-left-module of $A$. There exists a (separable semisimple) subalgebra $A_{0}$ of $A$ such that $A$ is the direct sum $A_{0} \oplus N$. As one readily sees from the argument of ground field extensions, we may, in order to prove our assertion, assume that $A_{0}(\cong A / N)$ is split, i.e. is a direct sum of mutually orthogonal matric algebras over $\Omega$, say $h$ in number. Let, under this assumption,

$$
\left\{e_{\mu \nu}^{(\kappa)} ; \mu, \nu=1,2, \ldots, n_{\kappa}\right\} \quad(\kappa=1,2, \ldots, h)
$$

be the $h$ systems of matric units in those matric algebras. Suppose now that $\mathfrak{I}$ is a singular extension of $A$ with kernel $\mathfrak{n}$; thus $\mathrm{n}^{2}=0$ and the residue-algebra

8) See Ikeda [4], Lemma 9. It is perhaps of use to note that here we do not need assume that $A$ is finite over $\Omega$ (as we see by modifying Ikeda's proof a little). 
$\mathfrak{T} / \mathfrak{n}$ is isomorphic to $A$. Let $\Re, \Re_{1}$ be the left-ideals of $\mathfrak{N}$ containing $\mathfrak{n}$ such that $\Re / \mathfrak{n}, \Re_{1} / \mathfrak{n}$ are mapped onto $N, N_{1}=\sum_{\kappa=1}^{h} N e_{11}^{(\kappa)}(\subseteq A)$, respectively, by our isomorphism. There are $h$ systems $\left\{e_{\alpha_{\nu}}^{(\kappa) *}\right\}$ of matric units in $\mathfrak{N}$ such that $e_{\mu \nu}^{(\kappa) *}$ $\bmod \mathfrak{n}$ corresponds to $e_{\mu \nu}^{(\kappa)}$, by our isomorphism. As $N_{1}$ is, together with $N$, an $\left(M_{0}\right)$-left-module of $A$, there exists a left- $\left(\mathfrak{R}^{-}\right)$submodule $N_{i}^{*}$ of $\mathfrak{R}_{1}$ such that $\Re_{1}$ is the direct sum $N_{1}^{*} \oplus \mathfrak{n}$. Put now $N^{*}=\sum_{\kappa=1}^{h} \sum_{\nu=1}^{n_{\kappa}} N_{\nu}^{*} e_{1 \nu}^{(\kappa) *}$. As $N=\sum_{\kappa=1}^{h} \sum_{\nu=1}^{n_{\kappa}} N_{1} e_{1 \nu}^{(\kappa)}$, clearly $\Re=N^{*}+\mathfrak{n}$. One sees further that the last sum is direct: $\Re=N^{*} \oplus \mathfrak{n} . N^{*}$ is a left-ideal of $\mathfrak{A}$ and is, further, $A_{0}^{*}$-right-allowable. Hence $\left(A_{0}^{*} \oplus N^{*}\right)^{2} \subseteq A_{0}^{* 2}$ $+N^{*}+N^{*} A^{*} \subseteq A^{*} \oplus N^{*}$, and $A_{0}^{*} \oplus N^{*}$ is a subalgebra of $\mathfrak{2}$. Since evidently $\mathfrak{N}=\left(A_{0}^{*} \oplus N^{*}\right) \oplus \mathfrak{n}, A$ is segregated in the extension $\mathfrak{l}$. Since $\mathfrak{H}$ is any singular extension of $A$, this proves our assertion.

Addendum: The main result of the present note has recently been extended, in a certain formulation, to algebras with vanishing cohomology groups in some dimensions higher than 2. This and further results on algebras with vanishing cohomology groups will be given in a joint paper to come by $M$. Ikeda, H. Nagao and the writer.

\section{REFERENCES}

[1] G. Hochschild, On the cohomology groups of an associative algebra, Ann. Math. 46 (1945), 58-6?.

[2] --, On the cohomology theory for associative algetras, Ann. Math. 47 (1646), 568-579.

[3] _ Cohomology and representations of associative algebras, Dure Math. J. 14 (1947), 921-948.

[4] M. Ikeda, On absolutely segregated algebras, Nagoya Math. J., this number.

[5] H. Nagao, Note on the cohomology groups of associative algebras, Nagoya Math. J., this number.

[6] H. Nagao-T. Nakayama, On the structure of $\left(M_{0}\right)$ - and $\left(M_{:}\right)$-modules, forthcoming in Math. Zeitschr.

Mathematical Institute, Nagoya University 